Ann. Biol. anim. Bioch. Biophys., 1977, 17 (2), 193-206

\title{
Application de quelques modèles de dynamique des populations à l'éfude de la folliculogenèse ovarienne
}

\author{
par J. C. MARIANA et C. MILLIER*
}

\author{
Station de Physiologie de la Reproduction, I. N. R. A. \\ Nouzilly, 37380, Monnaie, France \\ * Station de Biométrie, I. N. R. A. \\ 54370 Einville, France
}

Summary. Application of some population dynamics models to the sfudy of ovarian folliculogenesis.

We present two probability models for studying the renewal of growing follicles. The basic hypotheses for these two models are as follows :

1) All follicles having begun growth ovulate or become atretic,

2) a given follicle evolves independently from all the others,

3) the growth of a follicle and its dynamics are Markovian processes, depending on past events solely through the present situation.

A. - In the first model the growth of a given follicle is studied as a continuous phenomenon in time; the evolution of the number of growing follicles in time can be expressed by a differential equation. Either one or the other of the following factors is considered in characterizing a follicle : follicles ;

- age, estimated from the time the follicle leaves the pool of small primordial,

- biological parameter, typifying either follicle growth or metabolic activity.

B. - In the second model follicle growth is seen as a succession of maturation stages. The duration of each stage obeys the law of probability, and atresia in each stage is considered as an « accident », its probability of occurrence varying with the stage. Thus, we study the survival probability in time of a given follicle.

The second model is considered as a preliminary analytical step; the first is synthetic and should integrate the results of the second.

L'étude de la croissance et du renouvellement des follicules ovariens en est à un point où un certain formalisme est nécessaire pour avoir une vue globale et dégager des idées nouvelles de recherche analytique.

II ressort des travaux de Desaive (1947), Pedersen (1970), Mariana et Machado (1976) que le renouvellement de la population des follicules en croissance est assuré : 
- d'une part, par le recrutement de follicules à partir du stock des petits follicules ayant moins de 40 cellules folliculaires; ce stock, constitué chez les mammifères peu après la naissance, n'est plus renouvelé pendant la vie ;

- d'autre part, par des processus de dégénérescence qui peuvent survenir à tous les stades de la croissance du follicule et en outre chez les femelles adultes cycliques, par l'ovulation.

Par ailleurs, l'ensemble des régulations intra-ovariennes est encore inconnu (Desaive, 1947 ; Peters et al., 1973).

Notre but a donc été d'intégrer au niveau d'un modèle mathématique les deux mécanismes précédents qui assurent la permanence dans l'ovaire d'un certain nombre de follicules en croissance, d'en déduire la durée de la croissance et d'estimer les risques d'atrésie survenant au cours de cette même croissance.

De tels modèles ont été construits par Lotka (1907) pour décrire l'évolution de la démographie d'un pays au cours du temps, repris par von Foerster (1959), analysés et adaptés par Trucco (1965) à des problèmes de cinétique cellulaire.

Nous avons considéré deux types de modèle :

- le premier permet, au moyen d'équations appelées équations de renouvellement ou de diffusion, de suivre l'évolution au cours du temps de la population des follicules en croissance et plus particulièrement d'établir la structure de la population en fonction de l'âge des follicules ou d'un paramètre de croissance ou d'activité métabolique dépendant également du temps. La présentation des équations de renouvellement est déterministe, mais nous montrerons que le problème de la folliculogenèse ne peut être traitée qu'à partir d'un modèle aléatoire ou stochastique ;

- dans le second, la croissance d'un follicule est représentée comme une succession de stades dont la durée suit une distribution de probabilité ; on détermine alors la probabilité de survie d'un follicule au cours de sa croissance.

\section{A. - Modèle des équations de renouvellement.}

1. Renouvellement en fonction du temps et de l'âge des follicules.

a) Version déterministe.

Pedersen (1970) a établi la forme de la courbe de croissance d'un follicule en fonction du temps, en raccordant les courbes de croissance successives caractéristiques de chaque stade du développement d'un follicule (fig. 1).

Chacun de ces stades est défini à partir du nombre des cellules de la granulosa et, dans chaque stade, la croissance du follicule estimée par le nombre des cellules de la granulosa est exponentielle (Hartman ef Pedersen, 1970 ; Pedersen et Hartman, 1971) avec un taux de doublement $T_{D_{i}}$ des cellules caractéristique du stade considéré.

Si $n_{i}(a)$ est le nombre de cellules de la granulosa d'un follicule d'âge $a$, dans le stade $\mathrm{Si}$, on a donc :

$$
n_{i}(a)=n_{i}(o) \exp \left(\frac{a \cdot \ln 2}{T_{D 1}}\right)
$$


où $T_{D i}$ est le temps de doublement des cellules de la granulosa dans ce stade et $n_{j}(0)$ le nombre des cellules de la granulosa du follicule à son entrée dans le stade Si.

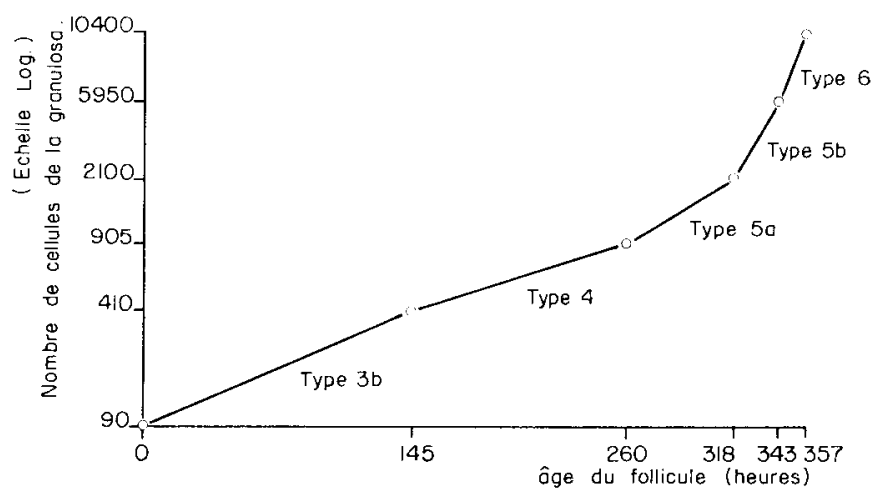

FIG. 1. - Courbe de croissance d'un follicule ovorien (partie de la croissance comprise entre 90 et 10400 cellules dans la granulosa) (d'après Pedersen, 1970).

Nous ferons essentiellement avec Pedersen l'hypothèse que la variation entre le nombre des cellules de la granulosa de follicules de même âge est faible par rapport au nombre moyen de ces mêmes cellules. Ainsi, il est possible, à partir de la connaissance de la courbe de croissance d'un follicule, d'établir une correspondance continue entre l'âge du follicule et le nombre des cellules de la granulosa. Afi.a da définir une dynamique des follicules à l'intérieur de l'ovaire, on doit complétar l'hypothèse précédente par les suivantes:

- Tout follicule ayant commencé sa croissance ovule ou devient atrétique ; il n'y a pas de mise au repos au cours de la croissance.

- La croissance ou la dégénérescence d'un follicule sont indépendants de la croissance ou de la dégénérescence des autres follicules.

La première hypothèse a été vérifiée ; en effet, quand on injecte à des souris ou à des rattes un précurseur radioactif de l'ADN, on observe que tous les follicules de l'ovaire sont marqués au niveau de la granulosa exceptés les primordiaux. La deuxième hypothèse n'a pas reçu de preuve à ce jour mais seulement des présomptions ; faute de meilleure, et sous réserve de confirmation expérimentale, on la conservera.

A la suite de Trucco (1965), nous définirons:

1) $\alpha(t) \mathrm{dt}$, le nombre des follicules qui entre $t$ et $t+d t$ ont reçu le signal de démarrer leur croissance.

2) $n(t, a) \Delta a$, le nombre des follicules survivant à l'instant t et dont l'âge est compris entre $a$ et $a+\Delta a$ et qui ont donc reçu le signal de démarrer lzur croissance à l'instant $(t-a)$.

$N(t)$, le nombre total des follicules normaux présents dans l'ovaire et qui ont déjà démarré leur croissance à l'instant t.

3) $\lambda(t, a)$, la proportion des follicules d'âge a qui deviennent atrétiques à l'instant $t$. 
La deuxième hypothèse spécifie que le taux d'atrésie $\lambda(t, a)$ ne dépend pas de la densité $n(t, a)$. Avec cette hypothèse, on peut donc écrire que le nombre des follicules d'âge a qui deviennent atrétiques entre $\dagger$ et $t+\Delta t$ est proportionnel à $n(t, a) \Delta a$, $\Delta t$ et $\lambda(t, a)$.

Le seul élément susceptible de modifier le nombre des follicules qui ont déjà commencé leur croissance étant l'atrésie, la variation du nombre total de follicules par unité de temps est donnée par :

$$
\frac{d N(t)}{d t}=\alpha(t)-\int_{0}^{a_{\max }} \lambda(t, a) \cdot n(t, a) \Delta a .
$$

Pendant un pelit intervalle de temps $t$, on peut écrire :

$$
n(t+d t, a+d t) \Delta a-n(t, a) \Delta a=-\lambda(t, a) n(t, a) \Delta t . \Delta a
$$

et en supposant les effectifs folliculaires suffisants pour justifier un passage à la limite, on montre que cette équation est équivalente à :

$$
\frac{\partial n(t, a)}{\partial \dagger}+\frac{\partial n(t, a)}{\partial a}=-\lambda(t, a) n(t, a) \text {. }
$$

Un schéma permet de visualiser le flux des follicules dans le temps (fig. 2).

Avec les hypothèses que nous avons présentées, il existe une solution unique :

$$
n(t, a)=\alpha(t-a) \cdot \exp \left(-\int_{0}^{a} \lambda(t-a+x, x) d x\right)
$$

Ainsi des $\alpha(t-a)$ dt qui ont démarré leur croissance à l'instant $(t-a)$, il reste $\mathrm{n}(\mathrm{t}, \mathrm{a}) \Delta \mathrm{a}$ follicules d'âge compris entre a et a $+\Delta \mathrm{a}$ à l'instant $\dagger$; dans l'expression de $n(t, a)$, l'exponentielle rend compte de l'aspect cumulatif des atrésies qui ont réduit le nombre des follicules au cours de leur croissance.

Précisons que cette présentation du modèle de Von Foerster ne peut servir qu'à exposer, d'une part, le problème de la dynamique folliculaire et à définir, d'autre part, les paramètres essentiels de cette dynamique car dans une perspective déterministe, on conçoit que les notions de passage à la limite ou de dérivée soient justifiées quand les effectifs des populations considérées sont importants (bactéries, insectes, etc...) ; or, le nombre des follicules en croissance dans un ovaire de ratte ou de brebis est compris entre 100 et 250 : il est donc faible dans une classe d'âge donnée et la dérivée n'a plus de sens.

II est cependant possible d'étendre le modèle précédent au cas de petites populations et d'aborder le problème de la variabilité biologique.

b) Version stochastique.

Kendall (1951) et Bartlett (1955) ont développé, généralisé et analysé les équations de renouvellement en considérant la croissance d'une population d'individus comme un processus aléatoire. L'hypothèse essentielle dans leur raisonnement est que le processus soit markovien ; très schématiquement, ceci signifie que l'histoire de la folliculogenèse d'une femelle donnée est résumée à un instant donné par la distri- 
bution des follicules dans l'ovaire en fonction de leur âge. (Pour une définition plus précise d'un processus markovien et de ses propriétés, cf. Karlin, 1969.) Par la méthode de la fonctionnelle caractéristique, Kendall aboutit à des équations correspondant à chacune des fonctions cumulantes, par exemple:

$$
\frac{\partial u}{\partial t}+\frac{\partial u}{\partial x}=-\mu u \operatorname{arec} E[d N(x, t)]=u(x, t) d x+o(d x)
$$

où $\int_{x_{1}}^{x_{2}} d N(x, t)$ est le nombre des individus qui à l'instant t occupent le groupe d'âge $\left(x_{1}, x_{2}\right), \mu(x) d t+$ odt est la probabilité qu'un individu d'âge $x$ vivant à l'instant $\dagger$ meure dans l'infervalle de temps suivant dt. E est l'opérateur espérance mathématique et $\mathrm{o}(\mathrm{dx})$ correspond aux termes infiniment petits d'ordre supérieur à 1.

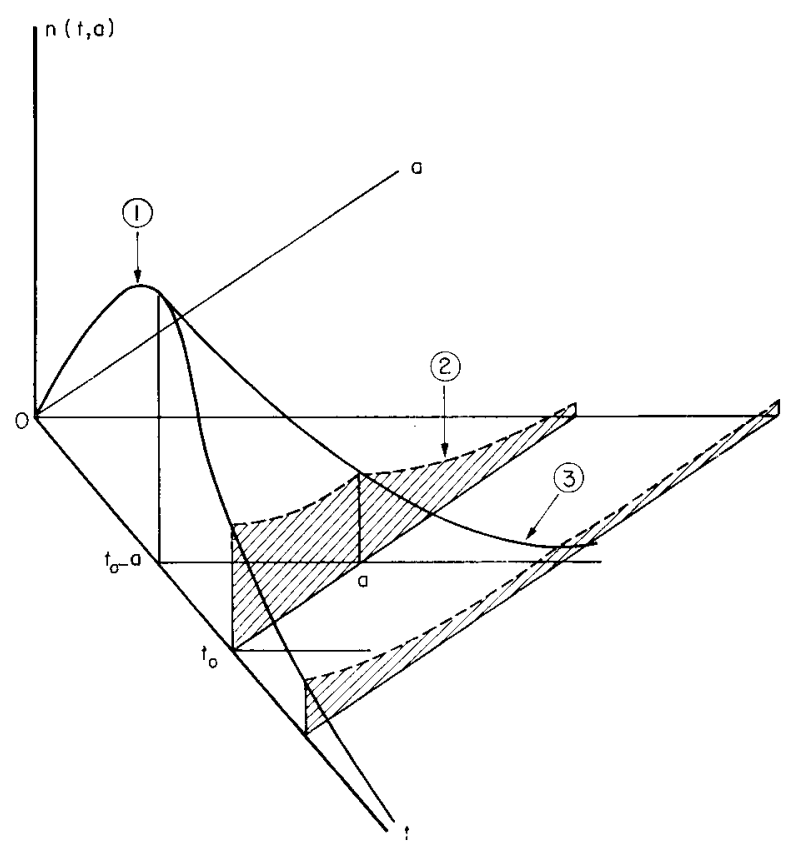

FIG. 2. Evolution de la population folliculaire dans un système de coordonnées où :

- a est l'âge du follicule,

- t le temps (l'origine $O$ correspond à la formation du 1 er follicule)

- $n(t, a)$ la fonction de densité du nombre des follicules survivants à l'instant $t$ et dont l'âge est compris entre $a$ et $a+\Delta a$

La courbe (1) contenue dans le plan $[t, n(t, a)]$ correspond au démarrage de la croissance des follicules au cours du temps.

La courbe (2) correspond à la distribution des follicules à un instant to en fonction de leur âge.

La courbe (3) contenue dans un plan parallèle au plan bissecteur du trièdre $0\{a, t, n(t, a)\}$ illustre l'évolution d'une cohorte de follicules qui ont démarré leur croissance à l'instant $t_{0}-a$; cette courbe est décroissante, car le nombre des follicules survivants diminue quand leur âge croît. 
Remarquons au passage que dans un processus de renouvellement (Bartlett, 1970 ), les éléments qui « tombent en panne » au cours de leur mise en service sont remplacés par des neufs; or, dans notre cas, il n'existe aucune preuve expérimentale (Peters et al., 1972) que le démarrage de la croissance de nouveaux follicules à partir du stock des primordiaux soit automatiquement contrôlé par l'atrésie (" la panne ») des follicules en cours de croissance, donc que l'on puisse écrire entre $\alpha(t)$ et $\lambda(t, a)$, la relation :

$$
\alpha(t)=\int_{0}^{a_{\max }} \lambda(t, a) n(t, a) d a .
$$

2. Renouvellement en fonction du temps et d'un indice de maturation des follicules.

a) Version déferministe.

- Age chronologique ef âge biologique.

La notion d'âge chronologique est, en fait, une notion commode car elle permef de dater et situer les phénomènes physiologiques les uns par rapport aux autres, mais elle est artificielle $\epsilon t$ on la connaît difficilement car il n'est pas toujours possible de "marqusr» les structures biologiques étudiées et de les suivre dans le temps comm气 l'ont fait Peters et Levy (1966) pour le follicule d'ovaire de souris. Par ailleurs, on conçoit mal que l'ovocyte cu les cellules folliculeuses aient la mémcire du temps : le follicule ovule quand il a atteint un niveau de maturation adéquat. Rübinow (1968) convient d'appeler «âge biologique » un ensemble de paramètres caractérisant un niveau de maturation.

Ces différents paramètres peuvent être le volume du follicule, le nombre des cellules de la granulosa, leur niveau de sécrétion ou tout autre caractérisant la croissance ou l'activité métabolique du follicule; chacune de ces variables est en ralation avec l'âge chronologique (fig. 3). Cette idée d'âge biologique a conduit Rübinow à développer un modèle déterministe du renouvellement d'une population où les paramètres sont le temps $\epsilon t$ un indice de maturation.

En conservant les hypothèses précédentes, on peut écrire encore une équation de variation du nombre total des follicules par unité de temps à l'instant $\uparrow$ :

$$
\frac{\mathrm{dN}(t)}{\mathrm{dt}}=\alpha(t)-\int_{0}^{\mathrm{M}} \lambda(t, \mu) \mathrm{n}(\mathrm{t}, \mu) \mathrm{d} \mu
$$

où l'âge biologique varie de 0 , pour un follicule qui démarre sa croissance, à $M$, valeur au-delà de laquelle aucun follicule ne survit.

L'équation de renouvellement sur laquelle nous n'insisterons pas est voisinc de celle de Von Foerster et pəut d'ailleurs s'y ramener après quelques manipulations : elle s'écrit :

$$
\frac{\partial n(t, \mu)}{\partial t}+\frac{\partial[\nu(t, \mu) \cdot n(t, \mu)]}{\partial \mu}=-\lambda(\mu) \cdot n(t, \mu)
$$

où $n(t, \mu) d \mu$ est le nombre des follicules dont l'indice de maturation à l'instant $t$ est compris entre $\mu$ et $\mu+d \mu$. 
$\lambda(\mu)$, le taux d'atrésie des follicules parvenus au niveau $\mu$ de maturation,

$\nu(t, \mu)$ est une vitesse de maturation des follicules ayant atteint le niveau de matu-

$$
\text { ration } \mu \text { à l'instant } t \text { avec } \nu=\frac{\partial \mu}{\partial t} \text { si } \mu=a, \nu=1 \text {. }
$$

b) Version stochastique.

A l'équation de renouvellement précédente, il correspond une version probabiliste valable pour les petites populations.

On introduit alors les définitions suivantes :

- $u(\mu, t)$ d l'espérance mathématique du nombre des individus qui ont atteint un niveau de maturation compris entre $\mu$ ef $\mu+d \mu$ à l'instant $t$.

- $E(\Delta \mu)$, espérance mathématique d'un petit changement $\Delta \mu$ du niveau de maturation $\mu$ pendant l'intervalle de temps $(t, t+d t)$ conditionnellement au fait que $\mu$ est déjà atteint, avec :

$$
E(\Delta \mu)=\nu(\mu, t) d t+o(d t)
$$

- $V(\Delta \mu)$ est la variance de ce même petit changement du niveau de maturation conditionnellement au fait que $\mu$ est déjà atteint.

L'hypothèse que le processus de la croissance d'un follicule ef celui du renouvellement des follicules en croissance sont des processus markoviens est essentielle pour la suite du raisonnement. On aboutit alors à des équations, appelées équations de diffusion, qui rendent compte de l'évolution d'une population d'individus en fonction du temps $t$ et d'un paramètre $\mu$ aléatoire de maturation.

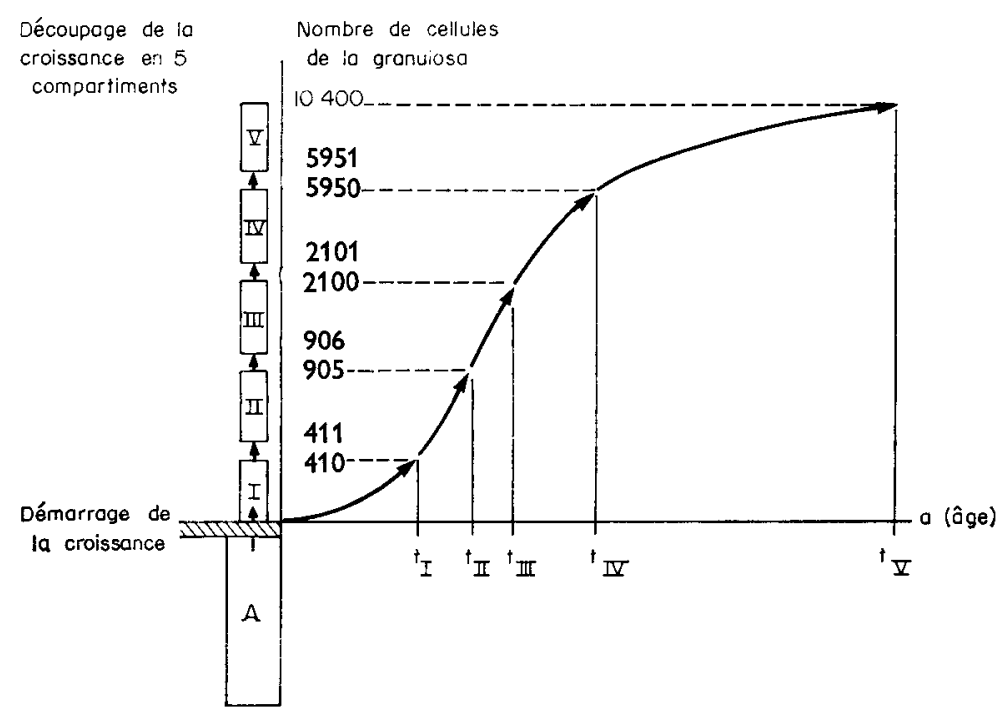

FIG. 3. - Schéma de découpage d'une croissance confinue en stades.

A correspond à la réserve des petits follicules. 
Conclusions sur les équations de renouvellement.

A l'issue de cette présentation succincte et simplifiée des équations de renouvellement, il semble que les modèles précédents permettent une représentation des mécanismes de la croissance et du renouvellement des follicules de l'ovaire. La croissance d'un follicule est envisagée comme un phénomène continu au cours du temps, mais le prix de cet effort de description est une complexité non négligeable des équations de diffusion et de leurs solutions.

Il est donc nécessaire, tout en conservant la notion d'âge physiologique et les avantages du modèle probabiliste, d'apporter quelques simplifications. Ainsi, une démarche voisine de la précédente consiste justement à étudier la probabilité conditionnelle qu'un follicule passe d'un niveau de maturation $\mu$ au niveau supérieur $\mu+d \mu$ dans l'intervalle de temps $(t, t+d t)$ conditionnellement au fait qu'il a déjà atteint le niveau $\mu$. La simplification consistera à découper la croissance d'un follicule en un certain nombre de stades consécutifs (fig. 3). En faisant des hypothèses sur la nature de la croissance ef de l'atrésie d'un follicule, il est possible d'estimer la durée passée par un follicule dans chacun des stades, à partir de données expérimentales.

\section{B. - Modèle multistade stochastique.}

Williams (1961) et Read et Ashford (1968) ont étudié l'évolution d'une population d'individus passant par plusieurs phases de croissance. Le modèle des derniers auteurs cités est suffisamment général pour permettre une description satisfaisante de la dynamique folliculaire.

La méthode consiste à analyser les probabilités de survie d'un individu au cours des différents stades de sa croissance.

La première étape consiste donc à découper la croissance d'un follicule en un certain nombre de stades, tels que ceux définis par Desaive (1947) et Pedersen et Peters (1968) ; on conçoit que la description de la croissance d'un follicule sera d'autant meilleure que le découpage de la croissance sera plus fin (fig. 3). Toutefois, un découpage trop fin risque d'affaiblir les effectifs dans chaque stade sans grand bénéfice (Pollard, 1966). Des études préliminaires sont donc nécessaires pour définir les largeurs optimums des classes d'âges biologiques ou des stades de maturation (Mariana et Machado, 1976).

Dans le modèle proposé par Read et Ashford, les hypothèses précédentes sont maintenues :

1) Tout follicule ayant commencé sa croissance ovule ou devient atrétique.

2) La croissance ou la dégénérescence d'un follicule est indépendante de celle des autres follicules.

Dans chaque stade $S_{i}$ de sa croissance, le follicule a une probabilité de devenir atrétique. Read et Ashford définissent ensuite une variable aléatoire $X_{1}$ minimum de deux autres variables aléatoires $Y_{i}$ et $Z_{i}$ attachées à chacun des stades de la croissance (cf. également Cox, 1966) :

- $Y_{i}$ est définie comme la durée du passage du follicule dans le stade $S_{1}$, sachant qu'il est normal quand il passe dans le stade $S_{1}+1$. 
- $Z_{i}$ est définie comme l'intervalle de temps séparant l'entrée du follicule dans le stade $S_{i}$ du moment où il devient atrétique dans ce stade.

L'atrésie est considérée comme un accident pouvant survenir à chaque stade, lequel est caractérisé par un taux d'atrésie $\mu_{\mathbf{i}}$.

On fait en outre l'hypothèse que la variable $Z_{i}$ suit une distribution exponentielle négative de densité $\mu_{\mathrm{i}} \mathrm{e}^{-\mu_{1} Z}$; une telle loi caractérise les arrivées d'accidents au hasard ; la variable $Y_{i}$ a une densité de probabilité $g_{i}(y)$ de même nature pour chaque stade mais de caractéristiques différentes.

Les auteurs considèrent plus particulièrement les distributions d'Erlang de densité :

$$
g_{i}(y)=\frac{\lambda_{i}^{a} \cdot y^{a-1}}{(a-1) !} e^{-\lambda_{i} y} \quad \text { (fig. 4) }
$$

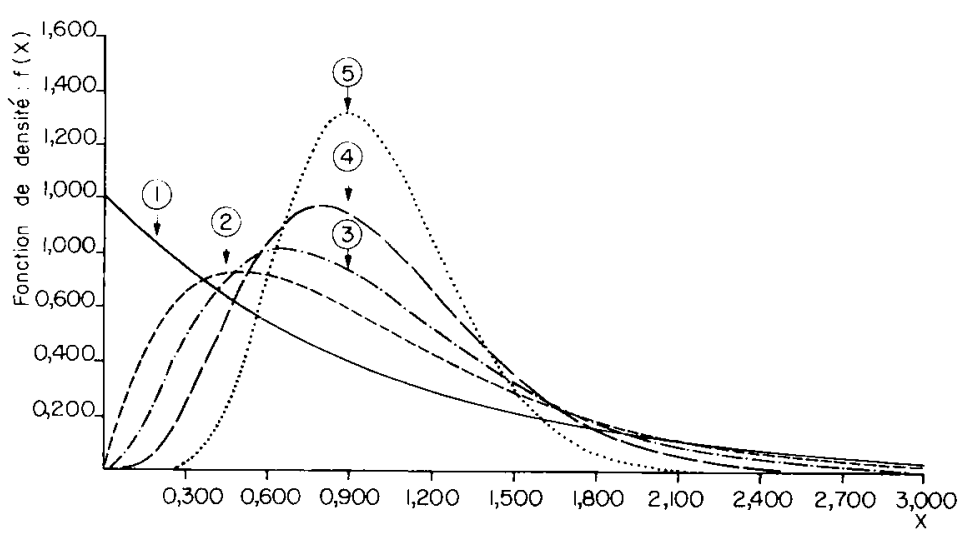

FIG. 4. - Quelques exemples de la loi d'Erlang $: f(x)=r \frac{(r x)^{a-1} \cdot e^{-r x}}{(a-1) !}$

$$
\text { de même moyenne : } m=1, m=\frac{a}{r}
$$

$$
\text { écart-type }: s=\frac{\sqrt{a}}{r}
$$

(1) $a=r=1$. Loi exponentielle négative.

(2) $a=r=2$.

(3) $a=r=3$.

(4) $a=r=4$.

(5) $a=r=5$.

Ces lois présentent des avantages mathématiques et on peut leur attacher la signification biologique suivante : le passage d'un niveau de maturation au suivant se fait en a étapes indépendantes de densité de probabilité $: \lambda_{1} \mathrm{e}^{-\lambda_{1} \mathrm{y}}(\mathrm{Cox}, 1966)$.

Les a étapes peuvent être par exemple des niveaux de maturation successifs de l'ovocyle du follicule dans le stade $i$ ou tout autres caractères. 
Si on fait l'hypothèse que $Y_{i}$ et $Z_{i}$ sont des variables aléatoires indépendantes, la densité de probabilité de $X_{\mathbf{i}}$ est alors :

$$
f_{i}(x) *=\mu_{i} e^{-\mu_{i} x} \cdot G_{i}(x)+g_{i}(x) \cdot e^{-\mu_{i} x}
$$

$\operatorname{avec}(x>0)$; en effet, un follicule demeure dans le stade i pendant une durée comprise entre $x$ et $x+d x$ de deux façons possibles :

1) Le stade $i$ a une durée supérieure à $x$ et le follicule devient atrétique un temps $x$ après son entrée dans le stade $i$ ( 1 er terme).

2) Le follicule survit pendant un intervalle de temps $x$ après son entrée dans le stade $i$ et passe dans le stade $i+1$ ( $2^{\mathrm{e}}$ terme).

- Après la décharge des gonadotropines, le follicule subit un ensemble de modifications importantes qui caractérisent le stade ultime du développement avant l'ovulation et on peut conserver pour la distribution de probabilité de la durée de ce stade, les mêmes types de lois que pour les autres stades.

- Le renouvellement des follicules est assuré par la réserve des follicules primordiaux et on peut considérer que cette réserve caractérise un stade $S_{0}$ initial et que les follicules passent de ce stade de repos $S_{0}$ au stade $S_{1}$ de croissance suivant une loi de probabilité identique à celle des autres słades, ou bien deviennent atrétiques avant de démarrer leur croissance.

- On peut prendre comme origine des temps de ce mécanisme le moment où le premier follicule démarre sa croissance dans un ovaire de jeune femelle.

En conservant le même type de raisonnement pour l'étude des follicules préovulatoires, on peut considérer comme origine des temps le moment du cycle où les hormones hypophysaires sont déchargées (Welschen, 1973).

Structure de la population folliculaire à un instant donné.

Le raisonnement de Read et Ashford (1968) reste complètement valable pour notre problème ; nous le reproduisons donc à ce titre.

La structure de la population folliculaire à un instant $\nmid$ donné s'établit à partir de la probabilité qu'un follicule choisi au hasard dans le stade Si soit normal à l'instant $\dagger$, soit $\mathrm{P}_{\mathbf{i}}(\mathrm{t})$ cette probabilité ; on définit la probabilité de passage d'un stade Si au suivant dans l'intervalle de temps $\left(u_{i}, u_{i}+\Delta u_{i}\right)$ et les fonctions de densité de probabilité du

$* \mathrm{e}^{-\mu_{\mathrm{i}} \mathrm{x}}=\int_{\mathrm{x}}^{\infty} \mu_{\mathrm{i}} \mathrm{e}^{-\mu_{\mathrm{i}} \mathrm{u}} \mathrm{du}$ est la fonction de survie de la loi exponentielle négative et

$$
G_{i}(x)=\int_{x}^{\infty} g_{i}(u) d u
$$

est la fonction de survie de la fonction $g_{i}(u)$.

On appelle fonction de survie $G(x)=$ Pro $(X>x)$ la probabilité qu'un individu n'ait pas eu de défaillance à l'instant $x: G(0)=1$ et $G(\infty)=0$. 
temps passé par un follicule dans un stade $\mathrm{Si}$, sachant qu'il est normal quand il va dans le stade $S(i+1)$ et conditionnellement au fait qu'il est entré dans le stade $S i$ :

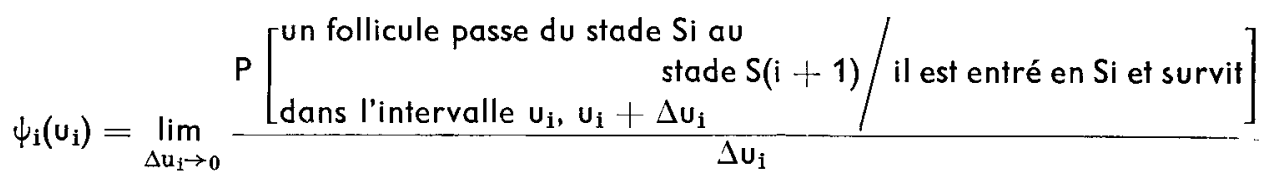

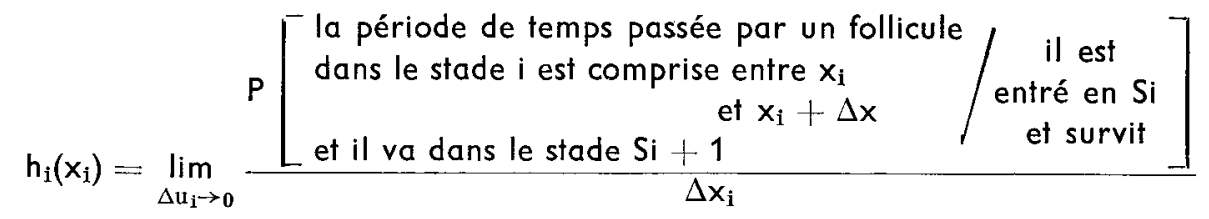

$\psi_{0}\left(u_{0}\right)$ est également la limite d'une probabilité qu'un follicule quitte le stade de repos $S_{0}$ et démarre sa croissance normalement à l'instant $v_{0}$ dans ce cas $\psi_{0}\left(u_{0}\right)=h_{0}\left(u_{0}\right)$ puisque $u_{0}$ est aussi la période passée par le follicule dans le stade $S_{0}$ depuis sa formation.

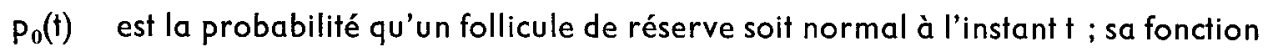
de survie $F_{0}(t)$ est donc égale à $p_{0}(t)$.

On a également : $p_{i}(t)=\int_{0}^{t} \psi_{i-1}\left(u_{i-1}\right) \cdot F_{i}\left(t-u_{i-1}\right) d u_{i-1}$ avec $F_{i}(x)$ fonction de survie au stade $i$.

On a défini précédemment la probabilité $f_{i}(x) d x$ qu'un follicule séjourne pendant un intervalle de temps compris entre $x$ et $x+d x$ dans le stade $S i, g_{i}(x) e^{-\mu_{i} x} d x$ est la probabilité que le follicule survive pendant un intervalle de temps compris entre $x$ et $x+d x$ dans le stade $i$ et passe dans le stade $S(i+1)$; donc $\frac{g_{i}(x) e^{-\mu_{i} x}}{f_{i}(x)}$ est la proportion de follicules qui passent dans le stade $i+1$ après être restés un temps compris entre $x$ et $x+d x$ dans le stade $i$.

On peut déduire tous les $\psi_{i}$ attachés à chaque stade, de proche en proche et toutes les probabilités $p_{i}(t) q u$ 'un follicule dans le stade $S i$ soit vivant à l'instant $t$ dans le stade Si conditionnellement au fait qu'il est entré dans ce stade.

Les auteurs, en utilisant des familles de loi d'Erlang, ont pu adapter leur modèle théorique aux données expérimentales.

La deuxième étape consiste donc à estimer les paramètres $\mu_{\mathrm{i}}$ caractérisant l'atrésie à chaque stade et les paramètres $\lambda_{i}$ (quand on s'est fixé le paramètre a des distributions erlangiennes).

C'est un problème d'estimation qui ne peut être développé qu'à partir d'un exemple.

\section{Discussion.}

La folliculogenèse est un processus de renouvellement particulier puisqu'au prix d'un gaspillage de ses stocks folliculaires, l'ovaire conduit à maturité un nombre réduit de follicules. 
Nous avons essayé de décrire avec un minimum d'arguments mathématiques deux modèles de renouvellement :

- Un premier modèle nous a conduit à définir des équations différentielles décrivant le flux des follicules en croissance d'un ovaire, au cours du temps, en fonction de leur âge ou d'un paramètre de maturation ; nous pensons que l'approche de la dynamique folliculaire par des équations probabilistes de renouvellement est la meilleure pour décrire les régulations intra-ovariennes des différentes catégories de follicules ef traduire des hypothèses biologiques, mais l'analyse de ces équations et l'ajustement à des données expérimentales restent difficiles. Il semble donc qu'on ne puisse les utiliser sous leur forme générale ; pour cette raison, nous proposons :

- Un second modèle, dans lequel on décrit la probabilité de survie d'un follicule au cours de son passage par plusieurs stades de croissance, permet, d'une part, une analyse directe et progressive de la population des follicules au cours du temps et, d'autre part, correspond mieux à ce que l'on connaît du phénomène de la croissance folliculaire.

II a le mérite de proposer une méthode d'étude de la croissance d'un élément d'une population qui évolue dans le temps, telle que la population folliculaire. On réalise ceci à partir d'un schéma d'échantillonnage et sur la base d'hypothèses réalistes et simples : chaque stade de la croissance d'un follicule a une durée aléatoire régie par une distribution de probabilité et le nombre des follicules qui deviennent atrétiques pendant un intervalle de temps suit une loi de Poisson de paramètre $\mu_{i}$ constant pour le stade considéré. On n'utilise donc pas de marquage radioactif du follicule ; ceci est un avantage incontestable puisqu'une telle utilisation est parfois impossible chez les gros animaux.

La méthode d'analyse des ovaires est de plus extrêmement simple puisqu'il s'agit pour chaque ovaire de dénombrer les follicules normaux dans les stades de croissance qu'on aura préalablement définis à partir des critères de classification disponibles (volume, affinités histochimiques, nombre de cellules, nombre de récepteurs...). Le dénombrement des follicules en début d'atrésie dans chaque stade est aisé si on admet qu'on peut considérer comme atrétiques les follicules ayant plus de 2 ou 3 cellules pycnotiques dans la granulosa.

Une difficulté immédiate surgit cependant au niveau de l'application car Read ef Ashford (1968) suivent la même population au cours du temps en réalisant des sondages, alors que dans notre cas chaque ratte représente une réalisation particulière d'un processus qui se déroule au cours du temps et qui est celui de la dynamique folliculaire ; il faut donc au niveau des paramètres $\lambda_{i}, \mu_{i}$ de chaque stade introduire des distributions d'échantillonnage el définir une stratégie d'estimation de ces différents paramètres.

Par ailleurs, il n'est pas prévu dans le modèle de Read ef Ashford (1968) que les paramètres $\lambda_{i}, \mu_{i}$ caractérisant chacun des stades varient avec le temps. Or, la vitesse de croissance ef les taux d'atrésie des follicules évoluent au cours de la vie et du cycle d'une femelle (Mariana et Nguyen Huy, 1973).

On sera donc conduit à découper la vie ou le cycle en un nombre de phases minimum tels qu'à l'intérieur de chacune les paramètres $\lambda_{i}$ et $\mu_{i}$ soient des constantes indépendantes du temps. 


\section{Conclusion.}

On doit reconnaître qu'aucun de ces modèles mathématiques décrivant la dynamique des populations n'est simple; la moindre connaissance biologique supplémentaire risque de les rendre encore plus complexes et parfois inutilisables, ce qui oblige à les expérimenter par approches successives.

Pour les raisons que nous avons développées antérieurement, il semble que le modèle de Read et Ashford soit une première étape possible dans une analyse pas à pas de la folliculogenèse. Une seconde étape consisterait à intégrer les hypothèses ef les résultats obtenus à partir du modèle précédent au niveau d'un modèle plus vaste : ce modèle est celui des régulations endocrines de la croissance des follicules au cours du cycle.

Une telle généralisation serait alors exprimée par des équations de renouvellement telles que celles qui ont été présentées.

\section{Accepté en octobre 1976.}

\section{Références}

BARTLETT M. S., 1955. Introduction to stochastic processes. Cambridge Univ. Press.

BARTLETT M. S., 1970. Age distributions. Biometrics, 26, 377-385.

COX D. R., 1966. Théorie du renouvellement. Traduit par J. LARRIEU, Dunod Ed.

DESAIVE P., 1947. Contribution à l'étude du mécanisme de l'évolution et de l'involution folliculaire dans l'ovaire de lapine adulte. Arch. Biol. 58, 331-446.

HARTMANN N. R., PEDERSEN T., 1970. Analysis of the kinetics of granulosa cell populations in the mouse ovary. Cell. Tissue Kinet, 3, 1-12.

KARLIN S., 1969. Initiation aux processus aléatoires. Traduit par Mme R. ROSTAND, Dunod Ed.

KENDALL D. G., 1951. Stochastic processes and population growth. J. R. Statisf. Soc. B, 11, 320-364.

LOTKA A., 1907. Studies on the mode of growth of material aggregates. Amer. J. Sci., 24, 199-216.

MARIANA J. C., 1970. Ełude sur le nombre d'ovulation obtenues après injection de PMSG chez la vache ; notion de seuil de réponse. Ann. Biol. anim. Bioch. Biophys., 10, 575-579.

MARIANA J. C., MACHADO J., 1976. Etude de la formation de l'antrum dans les follicules de l'ovaire de ratte et de vache normales ou stimulées par PMSG. Ann. Biol. anim. Bioch. Biophys., 16, 545-559.

MARIANA J. C., NGUYEN HUY N., 1973. Folliculogenèse chez la vache. Ann. Biol. anim. Bioch. Biophys., 13, hors série, 211-221.

PEDERSEN T., 1970. Determination of follicule growth rate in the ovary of the immature mouse. J. Reprod. Fert., 21, 81-93.

PEDERSEN T., HARTMANN N. R., 1971. The kinetics of granulosa cells in developing follicles in the mouse ovary. Cell. Tissue Kinet., 4, 171-184.

PEDERSEN T., PETERS H., 1968. Proposal for a classification of oocytes and follicles in the mouse ovary. J. Reprod. Fert., 17, 555-557.

PETERS H., LEVY E., 1966. Cell dynamics of the ovarian cycle. J. Reprod. Fert., 11, 227-236.

PETERS H., BYSKOV A. G., FABER M., 1973. Intraovarian regulation of follicle grcwth in the immature mouse 20-33. In PETERS $\mathrm{H}$. The development and maturation of the ovary and its functions. Excerpta Medica, Amsterdam.

POLLARD J. H., 1966. On the use of the direct matrix product in analysing certain stochastic population models. Biometrika, 53, 397-415.

READ K. L. Q., ASHFORD J. K., 1968. A system of models for the life cycle of a biological organism. Biometrika, 55, 211-221. 
RUBINOV S. I., 1968. A maturity time representation for cell populations. Biophys. J., 10, 1055-1073.

TRUCCO E., 1965. Mathematical models for cellular systems. The von Foerster Equation. Part I. Bull. Math. Biophys., 27, 285-304.

Von FOERSTER H., 1959. Some remarks on changing populations in the kinetics of cellular proliferation. Edit. by STOHLMAN F. Jr., N. Y., Grune \& Stratton, 382-407.

WELSCHEN R., 1973. Amounts of gonadotrophins required for normal follicular growth in hypophysectomized adult rat. Acta endocrinol., 72, 137-155.

WILLIAMS E. J., 1961. The growth and age distribution of a population of insects under uniform conditions. Biometrics, 17, 349-358. 